\title{
DETERMINAÇÃO SIMULTÂNEA DOS ÁCIDOS ORGÂNICOS TARTÁRICO, MÁLICO, ASCÓRBICO E CÍTRI- CO EM POLPAS DE ACEROLA, AÇAÍ E CAJU E AVALIAÇÃO DA ESTABILIDADE EM SUCOS DE CAJU
}

\author{
Rodrigo Scherer, Ana Cecília Poloni Rybka e Helena Teixeira Godoy* \\ Departamento de Ciência de Alimentos, Faculdade de Engenharia de Alimentos, Universidade Estadual de Campinas, CP 6121, \\ 13083-970 Campinas - SP, Brasil
}

Recebido em 16/7/07; aceito em 11/10/07; publicado na web em 2/4/08

\begin{abstract}
SIMULTANEOUS DETERMINATION OF TARTARIC, MALIC, ASCORBIC AND CITRIC ACIDS IN ACEROLA, AÇAI AND CASHEW PULPS, AND STABILITY EVALUATION IN CASHEW JUICES. The aim of the present study was determining the main organic acids in pulp and juices, as well as evaluating their stability, after opening the package, by liquid chromatography in a C18 column with isocratic elution and UV detection. In açaí pulp tartaric, malic and citric acids were found. Cashew samples presented all of the organic acids evaluated, besides high concentrations of ascorbic and malic acids. Acerola pulp had the highest ascorbic acid concentration. A small decrease in organic acid content during storage was observed. Malic and citric acids seem to be more stable than tartaric and ascorbic acids.
\end{abstract}

Keywords: HPLC; juice; vitamin C.

\section{INTRODUÇÃO}

As bebidas não alcoólicas têm largo consumo no mundo inteiro. Em todos os países, os levantamentos estatísticos revelam números crescentes de consumo, tanto per capita quanto global. Esses valores são mais expressivos quando se referem a bebidas obtidas de frutas, pelo fato das mesmas se constituírem em fontes fundamentais de vitaminas e minerais para a dieta humana, além de seus atrativos sabores. ${ }^{1}$

A indústria de polpas congeladas de frutas tem se expandido bastante nos últimos anos, notadamente no nordeste brasileiro. As unidades fabris se compõem, em sua maioria, de pequenos produtores que utilizam processos artesanais. A polpa congelada, por apresentar características de praticidade, vem ganhando grande popularidade, não só entre as donas de casa, mas também em restaurantes, hotéis, lanchonetes, hospitais, etc., onde é utilizada, principalmente, na elaboração de sucos. ${ }^{2}$

As frutas consistem em fonte nutricional de vitaminas, minerais e carboidratos solúveis, sendo que algumas possuem teor mais elevado de um ou de outro nutriente como, por exemplo, a acerola, que apresenta elevada quantidade de vitamina C. ${ }^{3}$ Esta vitamina é muito importante para a nutrição humana, sendo o ácido L-ascórbico sua principal forma biologicamente ativa. Como um antioxidante potente, tem a capacidade de eliminar diversos radicais livres. ${ }^{4}$

Além do ácido ascórbico, outros ácidos orgânicos estão extensamente distribuídos nas frutas e nos vegetais e sua concentração depende de fatores como espécie, solo e circunstâncias de estresse submetidas. ${ }^{5}$ Os ácidos orgânicos são também largamente utilizados como acidulantes na fabricação de bebidas à base de frutas e vegetais, sendo os principais ácidos utilizados para realçar sabores da bebida os ácidos cítrico e tartárico. Além disso, o ácido cítrico é muito utilizado como acidulante em sucos de fruta, porque o $\mathrm{pH}$ dado por ácidos naturais não é suficiente para assegurar a estabilidade microbiana a longo prazo. ${ }^{6}$

O ácido ascórbico é amplamente utilizado como antioxidante em sucos, entretanto, por causa de sua natureza, é oxidado durante

*e-mail: helena@fea.unicamp.br o período de estocagem. A literatura apresenta vários relatos sobre a estabilidade do ácido ascórbico em sucos de frutas, ${ }^{7-10}$ entretanto não há relatos sobre a estabilidade da vitamina $\mathrm{C}$, bem como dos demais ácidos orgânicos, em sucos após a embalagem ser aberta, já que o consumidor muitas vezes consome o suco durante alguns dias mantendo a embalagem aberta na geladeira, tornando-se importante tal avaliação.

A determinação precisa de nutrientes de frutas e vegetais é extremamente importante para se entender a relação entre o consumo e a saúde humana. Vários métodos têm sido relatados para avaliação de ácidos orgânicos, como espectrofotometria, ${ }^{11,12}$ enzimáticos, ${ }^{13}$ cromatografia gasosa, ${ }^{14}$ eletroforese capilar ${ }^{15,16}$ e titulação. ${ }^{17}$ Entretanto, a cromatografia líquida de alta eficiência tem sido a técnica mais empregada pela simplicidade, rapidez e estabilidade do método, bem como a dos compostos. ${ }^{6,18-25}$ Por essa razão, o objetivo do presente trabalho foi a avaliação simultânea dos principais ácidos orgânicos encontrados em caju, sucos de caju, polpa de caju, polpa de acerola e polpa de açaí, bem como a avaliação da estabilidade em suco de caju.

\section{PARTE EXPERIMENTAL}

As amostras de polpas de acerola, de açaí e de caju, suco de caju comercializado pronto para beber, suco de caju concentrado e caju (fruta) foram obtidas em três diferentes mercados em Campinas (São Paulo, Brasil) sendo avaliadas em três diferentes lotes. Cada lote consistiu de 3 itens (pacotes, garrafa ou fruta) homogeneizados.

Os padrões de ácidos orgânicos foram adquiridos da Supelco (USA) (Kit de ácidos orgânicos - 47264) e o fosfato de potássio monobásico da Merck (Alemanha). A água utilizada foi obtida do sistema Milli-Q (Millipore).

A determinação dos ácidos tartárico, málico, ascórbico e cítrico foi realizada em um cromatógrafo líquido HP 1100 series (Agilent) equipado com degaseificador, bomba quaternária, injetor automático ajustado para $20 \mu \mathrm{L}$ e detector de arranjo de diodos (DAD) ajustado a $250 \mathrm{~nm}$ para ácido ascórbico e a $210 \mathrm{~nm}$ para os demais ácidos, segundo $\mathrm{Facco}^{26}$ modificado. Para a separação 
Tabela 1. Parâmetros de validação do método para análise de ácidos orgânicos

\begin{tabular}{|c|c|c|c|c|c|c|c|c|c|c|}
\hline \multirow[t]{4}{*}{ Ácido } & \multicolumn{8}{|c|}{ Precisão } & \multirow[t]{2}{*}{ LD } & \multirow[t]{2}{*}{ LQ } \\
\hline & \multicolumn{3}{|c|}{ Repetibilidade } & & \multicolumn{4}{|c|}{ Precisão Intermediária } & & \\
\hline & \multicolumn{2}{|c|}{ nível 1} & \multicolumn{2}{|c|}{ nível 2} & \multicolumn{2}{|c|}{ nível 1} & \multicolumn{2}{|c|}{ nível 2} & & \\
\hline & *média & $\mathrm{CV}$ & média & $\mathrm{CV}$ & média & $\mathrm{CV}$ & média & $\mathrm{CV}$ & \multicolumn{2}{|c|}{$\mu \mathrm{g} / \mathrm{mL} \mu \mathrm{g} / \mathrm{mL}$} \\
\hline Tartárico & 23,93 & 0,41 & 2,34 & 1,58 & 23,91 & 1,59 & 2,35 & 1,19 & 0,72 & 1,20 \\
\hline Málico & 48,50 & 0,44 & 5,22 & 0,96 & 50,33 & 2,53 & 5,13 & 1,48 & 3,31 & 5,59 \\
\hline Ascórbico & 0,98 & 2,23 & 0,11 & 1,68 & 1,01 & 4,95 & 0,12 & 2,48 & 0,03 & 0,05 \\
\hline Cítrico & 40,16 & 0,51 & 3,61 & 1,91 & 39,04 & 2,28 & 3,57 & 2,07 & 1,87 & 3,09 \\
\hline
\end{tabular}

*mg $100 \mathrm{~mL}^{-1}$. CV: coeficiente de variação em \%; LD: limite de detecção; LQ: limite de quantificação.

cromatográfica foi utilizada uma coluna de fase reversa C18 (150 x 4,6 mm ID, $3 \mu \mathrm{m})$. A fase móvel utilizada para separação dos ácidos foi uma solução aquosa de $\mathrm{KH}_{2} \mathrm{PO}_{4} 0,01 \mathrm{M}$, com pH ajustado para 2,6 com ácido fosfórico na vazão de $0,5 \mathrm{~mL} / \mathrm{min}$. A identificação foi feita através dos tempos de retenção, espectros de absorção e co-cromatografia quando necessário. A quantificação foi realizada por curva de padronização externa com 7 pontos para cada ácido orgânico. As maiores concentrações dos padrões para elaboração das curvas foram 0,$25 ; 0,5 ; 0,01$ e $0,8 \mathrm{mg} \mathrm{mL}^{-1}$ para os ácidos tartárico, málico, ascórbico e cítrico, respectivamente, os outros seis pontos foram obtidos pela diluição em série com fase móvel (1/1). As curvas de calibração também foram utilizadas na avaliação da faixa de linearidade. Todas as amostras e a fase móvel foram filtradas em membrana 0,45 $\mu \mathrm{m}$ (Millipore JBR61022 e HAWP04700).

As amostras de polpas foram homogeneizadas em liquidificador comum com água, na proporção indicada na embalagem, diluídas com fase móvel (1/9), filtradas e injetadas no cromatógrafo. O suco concentrado foi preparado para consumo conforme descrito na embalagem e em seguida diluído com fase móvel (1/9), filtrado e injetado no cromatógrafo. $\mathrm{O}$ suco pronto para beber foi somente diluído com fase móvel (1/9), filtrado e injetado. A fruta foi prensada e o suco obtido foi diluído com fase móvel (1/9), filtrado e injetado no cromatógrafo.

A estabilidade dos ácidos orgânicos foi avaliada nos sucos de caju pronto para beber e concentrado após a embalagem ter sido aberta $(\mathrm{n}=3)$. O suco concentrado foi diluído em água segundo a recomendação do fabricante, e diluído novamente com fase móvel (1/9), filtrado e imediatamente injetado no cromatógrafo. Esse procedimento foi realizado em cada dia de análise. O suco pronto para beber foi diluído com fase móvel (1/9), filtrado e imediatamente injetado no cromatógrafo para cada dia de análise. As embalagens foram mantidas sob refrigeração a $5{ }^{\circ} \mathrm{C}$ e as análises realizadas nos dias zero (quando a embalagem foi aberta), 1, 4, 6, 8, 10 ,12 e 14 dias após a embalagem ter sido aberta.

Para validação do método foram avaliados os parâmetros de faixa de linearidade, precisão, exatidão, limite de detecção e limite de quantificação. A precisão envolveu a repetibilidade (10 injeções sucessivas, $n=10$ ) e a precisão intermediária ( 3 injeções em 3 dias diferentes na mesma semana, sendo este procedimento repetido após 6 meses, $\mathrm{n}=18$ ); ambos os parâmetros foram avaliados em dois níveis de concentração. A exatidão foi avaliada através da taxa de recuperação em dois níveis de concentração para cada ácido orgânico, em cada matriz. Os padrões de ácidos orgânicos foram adicionados nas amostras e a taxa de recuperação calculada como porcentagem de recuperação da quantidade adicionada. A quantidade naturalmente presente nas matrizes foi previamente determinada e dessa forma considerada na concentração final. Foram utilizados níveis de concentração, na validação do método, próximos aos níveis encontrados nas amostras. O limite da detecção foi considerado como a concentração mínima do analito cuja altura do pico foi de 3 vezes a amplitude do sinal de ruído, e 5 vezes para o limite de quantificação.

A análise estatística foi realizada utilizando o software Statistic 6.0. As diferenças foram consideradas significativas quando $P<$ 0,05 (ANOVA/teste de Tukey).

\section{RESULTADOS E DISCUSSÃO}

Os resultados obtidos para a validação do método estão apresentados nas Tabelas 1 e 2 . As faixas de linearidade avaliadas apresentaram coeficientes $\left(\mathrm{r}^{2}\right)$ de 1,$0 ; 1,0 ; 0,9998$ e 0,9995 para os ácidos tartárico, málico, ascórbico e cítrico, respectivamente. Os coeficientes de variação $(\mathrm{CV})$ para a repetibilidade variaram entre 0,4 e $2,3 \%$, e entre 1,2 a 5,0\% para a precisão intermediária, considerando os dois níveis avaliados. Valores maiores para a precisão intermediária se justificam pela realização das análises em diferentes dias e diferentes meses, entretanto os valores estão de acordo com os propostos por Horwitz et al. ${ }^{27}$ Os limites de detecção e quantificação demonstram a elevada sensibilidade do sistema, principalmente para o ácido ascórbico.

Tabela 2. Taxas de recuperação dos ácidos orgânicos nas matrizes avaliadas $(\%)$

\begin{tabular}{lcccc}
\hline & Tartárico & Málico & Ascórbico & Cítrico \\
\hline *Nível de adição 1 & 1 & 24 & 0,6 & 145 \\
& & & & \\
Suco pronto & 108 & 90 & 92 & 95 \\
Suco concentrado & 82 & 95 & 95 & 92 \\
Fruta & 82 & 86 & 92 & 86 \\
Polpas & $83-95$ & $85-98$ & $90-107$ & $85-94$ \\
& & & & \\
*Nível Adição 2 & 190 & 135 & 33 & 560 \\
& & & & \\
Suco Pronto & 99 & 97 & 99 & 96 \\
Suco concentrado & 86 & 98 & 97 & 98 \\
Fruta & 103 & 110 & 91 & 93 \\
Polpas & $90-101$ & $96-108$ & $90-97$ & $92-99$ \\
\hline
\end{tabular}

*mg $100 \mathrm{~mL}^{-1}$

As menores taxas de recuperação ocorreram nas polpas e frutas no menor nível de adição (Tabela 2). Ribani et al. ${ }^{28}$ relataram que a dispersão dos resultados aumenta com a diminuição da concentração adicionada e a recuperação pode diferir substancialmente em altas e baixas concentrações.

A Figura 1 apresenta um típico cromatograma obtido da separação de uma solução dos padrões (A) e um cromatograma obtido do suco de caju pronto para beber (B). Na Tabela 3 estão apresentados os resultados dos teores de ácidos orgânicos encontrados nas amostras avaliadas. O perfil de ácidos orgânicos encontrado nas amostras de caju foi muito semelhante, sendo que os menores va- 


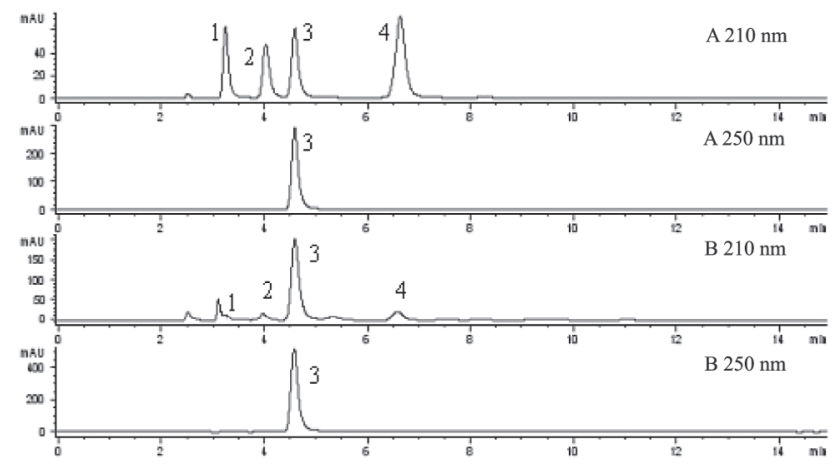

Figura 1. Perfil cromatográfico da solução padrão (A) e do suco de caju pronto para beber diluído com fase móvel (1/9) (B); 1 - ácido tartárico; 2 - ácido málico; 3 - ácido ascórbico; 4 - ácido cítrico. Condições cromatográficas: coluna de fase reversa C18 (150 × 4,6 mm ID, $3 \mu \mathrm{m})$ e fase móvel composta por solução aquosa de $\mathrm{KH}_{2} \mathrm{PO}_{4}$ 0,01 M, com pH ajustado para 2,6 com ácido fosfórico, vazão de 0,5 mL/min e leitura no detector a 210 e $250 \mathrm{~nm}$

lores para os ácidos orgânicos foram encontrados no suco pronto para beber e na polpa de caju. As amostras de suco de caju concentrado e suco puro da fruta de caju apresentaram valores muito semelhantes para os ácidos tártarico, málico e ascórbico, exceto para o acido cítrico que foi encontrado em grandes quantidades no suco concentrado devido à adição como acidulante.

Na polpa de açaí não foi encontrado ácido ascórbico, entretanto foram encontrados os ácidos málico, cítrico e pequena quantidade de ácido tartárico. O interesse pela fruta e polpa de açaí tem aumentado consideravelmente devido seu alto valor nutricional, principalmente pelo seu alto teor de antocianinas e poder antioxidante. ${ }^{29}$ Além disso, Galotta e Boaventura ${ }^{30}$ relataram atividade antitumoral de extratos de açaí. Há relatos de que a combinação de antocianinas com ácido ascórbico em presença do oxigênio causa perda de coloração, perda de propriedades funcionais e valor nutricional devido à degradação e polimerização de antocianinas monoméricas. ${ }^{31,32}$ Talvez por esse motivo o açaí não seja fonte de vitamina $\mathrm{C}$.

$\mathrm{Na}$ polpa de acerola foram encontradas grandes quantidades de ácido málico e ácido ascórbico, por outro lado, os ácidos tartárico e cítrico não foram encontrados (Tabela 3). Oliveira et al. ${ }^{33}$ avaliaram o teor de vitamina $\mathrm{C}$ em polpas de frutas congeladas, na polpa de caju foram encontrados valores entre 76 e $220 \mathrm{mg} 100 \mathrm{~g}^{-1}$, enquanto que na polpa de acerola os valores variaram entre $470 \mathrm{e}$

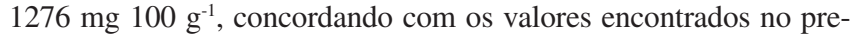
sente trabalho.

Na Tabela 4 estão apresentados os resultados da avaliação da estabilidade dos ácidos orgânicos nos sucos de caju concentrado e pronto para beber. Segundo os fabricantes, o suco pronto para beber deve ser consumido em até 3 dias após abertura da embalagem, enquanto que o suco concentrado em até 10 dias. Ao longo dos 14 dias de avaliação não houve diferença significativa nos teores de ácido málico e cítrico em ambos os sucos avaliados. Entretanto, o teor de ácido tartárico foi reduzido significativamente $(P<0,05)$ a partir do quarto dia no suco pronto para beber, e a partir do oitavo dia no suco concentrado (Tabela 4). O teor de ácido ascórbico teve uma redução significativa $(P<0,05)$ de $10 \%$ no suco concentrado de caju após 8 dias da embalagem ter sido aberta. No suco pronto para beber houve uma redução significativa $(P<0,05)$ após 12 dias de armazenagem com a embalagem aberta, entretanto, o valor encontrado ainda está acima do valor declarado pelo fabricante.

Segundo a resolução RDC no $360 / 03$ da ANVISA, ${ }^{34}$ item 3.5.1. do Regulamento Técnico Sobre Rotulagem Nutricional de Alimentos Embalados, a variação máxima dos nutrientes declarados no

Tabela 3. Ácidos orgânicos encontrados nas amostras analisadas

\begin{tabular}{|c|c|c|c|c|c|}
\hline Amostra & Lote & Tartárico & Málico & Ascórbico & Cítrico \\
\hline & & Média \pm SD & Média \pm SD & Média \pm SD & Média \pm SD \\
\hline \multirow{3}{*}{$\begin{array}{l}\text { Suco de Caju } \\
\text { pronto para beber } \\
\left(\mathrm{mg} 100 \mathrm{~mL}^{-1}\right)\end{array}$} & 1 & $4,43 \pm 0,73$ & $32,44 \pm 3,70$ & $47,42 \pm 2,25$ & $206,13 \pm 7,40$ \\
\hline & 2 & $4,30 \pm 0,34$ & $29,02 \pm 0,23$ & $50,19 \pm 0,94$ & $251,51 \pm 6,70$ \\
\hline & 3 & $4,53 \pm 0,46$ & $35,86 \pm 2,64$ & $44,65 \pm 1,40$ & $238,44 \pm 14,86$ \\
\hline \multirow{3}{*}{$\begin{array}{l}\text { Suco de Caju } \\
\text { concentrado } \\
\left(\mathrm{mg} 100 \mathrm{~mL}^{-1}\right)\end{array}$} & 1 & $27,14 \pm 2,66$ & $208,61 \pm 10,71$ & $153,26 \pm 9,06$ & $529,14 \pm 9,54$ \\
\hline & 2 & $27,21 \pm 2,24$ & $235,67 \pm 5,09$ & $145,66 \pm 3,37$ & $434,91 \pm 12,73$ \\
\hline & 3 & $21,54 \pm 1,36$ & $237,90 \pm 3,46$ & $161,36 \pm 8,70$ & $578,80 \pm 16,56$ \\
\hline \multirow{3}{*}{$\begin{array}{l}\text { Caju } \\
\left(\mathrm{mg} 100 \mathrm{~mL}^{-1}\right)\end{array}$} & 1 & $32,72 \pm 3,89$ & $293,21 \pm 28,33$ & $186,42 \pm 0,59$ & $8,97 \pm 0,75$ \\
\hline & 2 & $26,21 \pm 0,54$ & $200,96 \pm 15,04$ & $165,61 \pm 2,55$ & $7,67 \pm 0,61$ \\
\hline & 3 & $29,03 \pm 2,04$ & $198,85 \pm 7,32$ & $155,37 \pm 4,22$ & $9,89 \pm 0,38$ \\
\hline \multirow{3}{*}{ 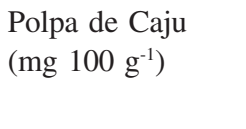 } & 1 & $16,84 \pm 1,41$ & $103,80 \pm 4,40$ & $114,41 \pm 1,00$ & $6,47 \pm 1,05$ \\
\hline & 2 & $18,05 \pm 0,87$ & $105,58 \pm 5,95$ & $109,93 \pm 1,94$ & $5,67 \pm 0,64$ \\
\hline & 3 & $17,79 \pm 0,72$ & $104,49 \pm 2,92$ & $115,76 \pm 1,37$ & $6,27 \pm 0,46$ \\
\hline \multirow{3}{*}{$\begin{array}{l}\text { Polpa de Açaí } \\
\left(\mathrm{mg} 100 \mathrm{~g}^{-1}\right)\end{array}$} & 1 & $4,34 \pm 0,19$ & $34,89 \pm 1,85$ & $\mathrm{Nd}$ & $30,58 \pm 4,91$ \\
\hline & 2 & $3,72 \pm 0,19$ & $36,17 \pm 5,81$ & $\mathrm{Nd}$ & $26,70 \pm 1,49$ \\
\hline & 3 & $3,68 \pm 0,26$ & $51,85 \pm 1,30$ & $\mathrm{Nd}$ & $30,43 \pm 1,72$ \\
\hline \multirow{3}{*}{$\begin{array}{l}\text { Polpa de Acerola } \\
\left(\mathrm{mg} 100 \mathrm{~g}^{-1}\right)\end{array}$} & 1 & $\mathrm{Nd}$ & $344,54 \pm 55,30$ & $494,07 \pm 65,95$ & $\mathrm{Nd}$ \\
\hline & 2 & $\mathrm{Nd}$ & $411,87 \pm 33,81$ & $441,12 \pm 26,91$ & $\mathrm{Nd}$ \\
\hline & 3 & $\mathrm{Nd}$ & $418,65 \pm 9,08$ & $468,91 \pm 18,13$ & $\mathrm{Nd}$ \\
\hline
\end{tabular}

Nd: Não detectado 
Tabela 4. Estabilidade dos ácidos orgânicos em sucos de caju sob refrigeração após a embalagem ser aberta $\left(\mathrm{mg}^{100} \mathrm{~mL}^{-1}\right)$

\begin{tabular}{|c|c|c|c|c|c|c|c|c|}
\hline \multirow[b]{2}{*}{ Dias } & \multicolumn{4}{|c|}{ Suco concentrado } & \multicolumn{4}{|c|}{ Suco pronto para beber } \\
\hline & tartárico & málico & ascórbico & Cítrico & tartárico & málico & ascórbico & cítrico \\
\hline 0 & $27,2 \mathrm{a}$ & $236,1 \mathrm{a}$ & $145,6 a$ & $470,8 \mathrm{a}$ & $4,3 \mathrm{a}$ & $29,0 \mathrm{a}$ & $50,1 \mathrm{a}$ & $231,1 \mathrm{a}$ \\
\hline 1 & $27,4 a$ & $236,6 \mathrm{a}$ & $150,2 \mathrm{a}$ & $470,5 \mathrm{a}$ & $4,3 a$ & $25,7 \mathrm{a}$ & $50,5 \mathrm{a}$ & $232,2 a$ \\
\hline 4 & $25,6 \mathrm{a}$ & $236,1 \mathrm{a}$ & $153,0 \mathrm{a}$ & $471,8 \mathrm{a}$ & $3,8 \mathrm{a}$ & $27,1 \mathrm{a}$ & $50,0 \mathrm{a}$ & $234,3 a$ \\
\hline 6 & $25,9 \mathrm{a}$ & $237,3 \mathrm{a}$ & $145,0 \mathrm{a}$ & $483,0 \mathrm{a}$ & $3,2 b$ & $27,3 \mathrm{a}$ & $50,7 \mathrm{a}$ & $233,8 a$ \\
\hline 8 & $19,2 b$ & $236,1 \mathrm{a}$ & $133,9 b$ & $474,5 \mathrm{a}$ & $2,7 \mathrm{c}$ & $27,0 \mathrm{a}$ & $49,2 \mathrm{a}$ & $233,7 a$ \\
\hline 10 & $20,1 b$ & $235,3 a$ & $132,1 \mathrm{~b}$ & $476,4 \mathrm{a}$ & $2,1 \mathrm{~d}$ & $27,6 a$ & $48,9 a$ & $234,3 a$ \\
\hline 12 & $19,8 b$ & $236,1 \mathrm{a}$ & $140,5 b$ & $485,7 \mathrm{a}$ & $2,6 \mathrm{~d}$ & $27,5 \mathrm{a}$ & $48,0 \mathrm{~b}$ & $232,1 \mathrm{a}$ \\
\hline 14 & $15,0 \mathrm{c}$ & $236,7 \mathrm{a}$ & $137,1 \mathrm{~b}$ & $481,3 \mathrm{a}$ & $2,2 d$ & $27,1 \mathrm{a}$ & $48,3 b$ & $232,8 \mathrm{a}$ \\
\hline
\end{tabular}

Letras diferentes na mesma coluna correspondem a diferenças significativas $(P<0,05)$.

rótulo permitida é de $20 \%$ a mais que o declarado pelo fabricante. No suco de caju pronto para beber foi declarada no rótulo a quantidade de $11,5 \mathrm{mg} 100 \mathrm{~mL}^{-1}$ de ácido ascórbico, entretanto foi encontrada cerca de 4 vezes a mais (Tabela 3). Castro et al. ${ }^{9}$ relataram resultados semelhantes na avaliação de vitamina $\mathrm{C}$ em bebidas isotônicas, onde foram encontrados níveis de vitamina $\mathrm{C}$ acima do declarado pelos fabricantes. Nas embalagens das frutas de caju foram declarados 139-387 mg $100 \mathrm{~g}^{-1}$, estando de acordo com os valores encontrados (Tabela 3). No restante das amostras não foi declarado o teor de vitamina $\mathrm{C}$.

Ingestão Diária Recomendada (IDR) é a quantidade de um determinado nutriente que deve ser consumida diariamente para atender às necessidades nutricionais da maior parte dos indivíduos e grupos de pessoas de uma população sadia. De acordo com a legislação brasileira ${ }^{35}$ e Food and Agriculture Organization, ${ }^{36}$ a IDR para o ácido ascórbico em um adulto é de 60 e $45 \mathrm{mg}$, respectivamente. Sendo assim, o consumo de aproximadamente $200 \mathrm{~mL}$ (um copo) de qualquer amostra avaliada, mesmo considerando as diluições propostas pelos fabricantes, é suficiente para suprir a IDR de vitamina $\mathrm{C}$, exceto a polpa de açaí na qual não foi encontrada essa vitamina.

\section{CONCLUSÕES}

O método desenvolvido mostrou-se rápido e eficiente na avaliação simultânea dos ácidos orgânicos tartárico, málico, ascórbico e cítrico, além disso, o método gerou pouco resíduo e não tóxico. Dentre as amostras avaliadas a acerola apresentou o maior teor de vitamina $\mathrm{C}$, entretanto, com exceção da polpa de açaí, todas as amostras avaliadas são boas fontes de vitamina $C$, sendo necessário o consumo de menos de $200 \mathrm{~mL}$ para suprir a ingestão diária recomendada para essa vitamina. O suco de caju apresentou moderados teores de ácido málico, que talvez possa ser utilizado como parâmetro de qualidade em sucos naturais de caju, pois em preparados artificiais não é comum encontrar esse ácido. Não foi verificada nenhuma alteração nos teores dos ácidos málico e cítrico durante a armazenagem, mesmo com a embalagem aberta, entretanto, os ácidos tartárico e ascórbico foram reduzidos durante o período de armazenagem.

\section{REFERÊNCIAS}

1. Soares, L. C.; Oliveira, G. S. F.; Maia, G. A.; Monteiro, J. C. S.; Junior, A. S.; Rev. Bras. Frutic. 2001, 23, 387.

2. Oliveira, M. E. B.; Bastos, M. S. R.; Feitosa, T.; Branco, M. A. A. C.; Silva, M. G. G.; Ciênc. Tecnol. Aliment. 1999, 19, 326.
3. Matsuura, F. C. A. U.; Rolim, R. B.; Rev. Bras. Frutic. 2002, 24, 138.

4. Davey, M. W.; van Montagu, M.; Inzé, D.; Sanmartin, M.; Kanellis, A.; Smirnoff, N.; Benzie, I. J. J.; Strain, J. J.; Favell, D.; Fletcher, J.; J. Sci. Food Agr. 2000, 80, 825.

5. Jones, D. L.; Plant Soil. 1998, 205, 25.

6. Shui, G.; Leong, L. P.; J. Chromatogr., A. 2002, 977, 89.

7. Zerdin, K.; Rooney, M. L.; Vermue, J.; Food Chem. 2003, 82, 387.

8. Leizerson, S.; Shimoni, E.; J. Agric. Food. Chem. 2005, 53, 4012.

9. de Castro, F. J.; Scherer, R.; Godoy, H. T.; Quim. Nova 2006, 29, 719.

10. Ros-Chumillas, M.; Belisario, Y.; Iguaz, A.; López, A.; J. Food. Eng. 2007, 79, 234.

11. Rebelein, H.; Deut. Lebensm-Rundsch. 1961, 57, 6.

12. Vereda, E.; de Torres, A. G.; Rivero, A.; Cano, J. M.; Quim. Anal. 1998, $17,167$.

13. Puchades, R.; Herrero, M. A.; Maquieira, A.; Atienza, J.; Food Chem. 1991, 42, 167.

14. Cocchi, M.; Durante, C.; Grandi, M.; Lambertini, P.; Manzini, D.; Marchetti, A.; Talanta 2006, 69, 1166.

15. Cortacero-Ramírez, S.; Segura-Carretero, A.; de Castro, M. H. B.; Fernández-Gutiérrez, A.; J. Chromatogr., A 2005, 1064, 115.

16. Mato, I.; Huidobro, J. F.; Simal-Lozano, J.; Sancho, M. T.; Anal. Chim. Acta 2006, 565, 190.

17. AOAC; Official methods of analysis of the Association of Official Analytical Chemists, $15^{\text {th }}$ ed.; Arlington, VA, 1990.

18. Wang, T.; Gonzalez, A. R.; Gbur, E. E.; Aselage J. M.; J. Food Sci. 1993, 58,631 .

19. Lian, H. Z.; Mao, L.; Ye, X. L.; Miao, J.; J. Pharm. Biomed. Anal. 1999, 19,621 .

20. Cawthray, G. R.; J. Chromatogr., A 2003, 1011, 233.

21. Kerem, Z.; Bravdo, B.; Shoseyov, O.; Tugendhaft, Y.; J. Chromatogr., A 2004, 1052, 211.

22. Kotani, A.; Miyaguchi, Y.; Tomita, E.; Takamura, K.; Kusu, F.; J. Agric. Food. Chem. 2004, 52, 1440.

23. Destandau, E.; Vial, J.; Jardy, A.; Hennion, M. C.; Bonnet, D.; Lancelin, P.; J. Chromatogr., A 2005, 1088, 49.

24. Fontannaz, P.; Kilinc, T.; Heudi, O.; Food Chem. 2006, 94, 626.

25. Hernández, Y.; Lobo, M. G.; González, M.; Food Chem. 2006, 96, 654.

26. Facco, E. M. P.; Tese de Doutorado, Universidade Estadual de Campinas, Brasil, 2006.

27. Horwitz, W.; Kamps, L. R.; Boyer, K. W.; Quality Control. 1980, 63, 1344.

28. Ribani, M.; Bottoli, C. B. G.; Collins, C. H.; Jardin, I. C. S. F.; Melo, L. F. C.; Quim. Nova 2004, 27, 771.

29. Coisson, J.; Travaglia, F.; Piana, G.; Capasso, M.; Arlorio, M.; Food Res. Int. 2005. 38, 893

30. Galotta, A. L. Q. A.; Boaventura, M. A. D.; Quim. Nova 2005, 28, 610.

31. Garcia-Viguera, C.; Bridle, P.; Food Chem. 1999, 64, 21.

32. Pacheco-palencia, L. A.; Hawken, P.; Talcott, S. T.; Food Res. Int. 2007, 40,620 .

33. Oliveira, M. E. B.; Bol. CEPPA 1998, 16, 13.

34. http://www.anvisa.gov.br/alimentos/legis/especifica/rotuali.htm, acessada em Julho 2007.

35. http://www.anvisa.gov.br/alimentos/legis/especifica/regutec.htm, acessada em Março 2007.

36. http://www.fao.org/DOCREP/004/Y2809E/y2809e00.htm\#Contents, acessada em Março 2007. 